MATHEMATICS OF COMPUTATION

Volume 81, Number 277, January 2012, Pages 267-276
S 0025-5718(2011)02492-0

Article electronically published on June 20, 2011

\title{
ON A NEW CLASS OF ADDITIVE (SPLITTING) OPERATOR-DIFFERENCE SCHEMES
}

\author{
PETR N. VABISHCHEVICH
}

\begin{abstract}
Many applied time-dependent problems are characterized by an additive representation of the problem operator. Additive schemes are constructed using such a splitting and are associated with the transition to a new time level on the basis of the solution of more simple problems for the individual operators in the additive decomposition. We consider a new class of additive schemes for problems with additive representation of the operator at the time derivative. In this paper we construct and study the vector operator-difference schemes, which are characterized by a transition from the single initial evolution equation to a system of evolution equations.
\end{abstract}

\section{INTRODUCTION}

For the approximate solution of multidimensional unsteady problems of mathematical physics there are widely used different classes of additive schemes (splitting schemes) [7, 10,23. Beginning with the pioneering works $[2,8$ the simplest way to construct additive schemes is in the splitting of the problem operator on the sum of two operators with a simpler structure - alternating direction methods, factorized schemes, predictor-corrector schemes, etc. [14.

In the more general case of multicomponent splitting, classes of unconditionally stable operator-difference schemes are based on the concept of summarized approximation. In this way, we can construct the classic locally one-dimensional schemes (componentwise splitting schemes) [7,10, additively averaged locally onedimensional schemes [5, 14].

A new class of unconditionally stable schemes - vector additive schemes (multicomponent alternating direction method schemes) is actively developed (see, e.g., [1,20]). They belong to a class of full approximation schemes - each intermediate problem approximates the original one. The simplest additive full approximation schemes are based on the principle of regularization of operator-difference schemes. Improving the quality of operator-difference schemes is achieved using additive or multiplicative perturbations of operators of the scheme 9. Regularized additive schemes for evolutionary equations of the first and second order are constructed for equations as well as systems of equations [15,21]. First, the standard schemes of splitting with respect to separate directions (locally one-dimensional schemes)

Received by the editor May 12, 2010 and, in revised form, September 7, 2010.

2010 Mathematics Subject Classification. Primary 65M06, 65M12.

Key words and phrases. Evolutionary problems, splitting schemes, the stability of operatordifference schemes, vector additive schemes.

(C)2011 American Mathematical Society Reverts to public domain 28 years from publication 
and splitting with respect to physical processes are considered [7,10]. Second, regionally additive schemes based on domain decomposition for constructing parallel algorithms are studied for transient problems of mathematical physics [6, 12,22].

Our interest here is with mathematical models based on boundary value problems for equations of Sobolev's type. In his work [16] the following equation

$$
\frac{\partial^{2}}{\partial t^{2}}\left(\frac{\partial^{2} u}{\partial x_{1}^{2}}+\frac{\partial^{2} u}{\partial x_{2}^{2}}+\frac{\partial^{2} u}{\partial x_{3}^{2}}\right)+\alpha^{2} \frac{\partial^{2} u}{\partial x_{3}^{2}}=0,
$$

has been obtained for describing oscillations in a rotating liquid. Models with the time derivative of first order have been much studied for the pseudo-parabolic equation

$$
M \frac{d u}{d t}+L u=0
$$

where $M$ and $L$ are elliptic operators [17, 18. The most comprehensive review of applied problems connected with equations of Sobolev type is presented in the recently published book [19].

Starting from pioneer works [3, 4, many numerical algorithms have been developed for equations of Sobolev type. At present, different classes of additive operator-difference schemes for evolutionary equations are constructed via additive splitting of the operator $L$ (connected with the solution $u$ ) onto several terms [7, 14. For a number of applications it is more interesting to consider problems in which the additive representation takes place for the operator at the time derivative (operator $M)$. We have such a situation, for instance, for the above-mentioned Sobolev equation where the operator at the time derivative is the sum of three one-dimensional operators and the operator at $u$ is one-dimensional, too. The main advantage of the additive (splitting) schemes is associated with the solution of simpler problems for the individual operator terms at the transition to a new time level.

In this work, for this new class of evolutionary problems, the vector additive operator-difference schemes are constructed and studied. The work is organized as follows. Section 1 provides a statement of the problem along with a simple a priori estimate of the stability for the solutions with respect to initial data and righthand side. This estimate is just our reference point when considering the vector problem and the operator-difference schemes. The vector differential problem is considered in Section 2. The central part of the work (Section 3) deals with the construction and investigation of the stability of vector additive schemes. Possible generalizations of the results are discussed in Section 4.

\section{Statement of the Problem}

Let $H$ be a finite-dimensional Hilbert space, and let $A, B, D$ be linear operators in $H$. We consider grid functions $y$ of finite-dimensional real Hilbert space $H$, for the scalar product and norm in which we use the notations: $(\cdot, \cdot),\|y\|=(y, y)^{1 / 2}$. For $D=D^{*}>0$ we introduce the space $H_{D}$ with scalar product $(y, w)_{D}=(D y, w)$ and norm $\|y\|_{D}=(D y, y)^{1 / 2}$.

In the Cauchy problem for evolutionary equations of first order we search the function $y(t) \in H$, which satisfies the equation

$$
B \frac{d u}{d t}+A u=f(t), \quad t>0
$$


and the initial condition

$$
u(0)=u^{0}
$$

at a given $f(t) \in H$.

We assume that linear operators $A$ and $B$, acting from $H$ into $H(A: H \rightarrow H$, $B: H \rightarrow H)$, are positive, self-adjoint and stationary; that is,

$$
A=A^{*}>0, \frac{d}{d t} A=A \frac{d}{d t}, \quad B=B^{*}>0, \frac{d}{d t} B=B \frac{d}{d t} .
$$

For problem (1.1), (1.2) we can obtain different a priori estimates, which express the stability of the solution with respect to the initial data and right-hand side in different spaces. We restrict ourselves to the simplest of them, trying to get the same type of estimates for both the scalar and vector problems as well as for the solution of both differential and difference problems.

Scalarly multiplying both sides of equation (1.1) in $H$ by $u$, we get

$$
\frac{1}{2} \frac{d}{d t}(B u, u)+(A u, u)=(f, u)
$$

For the right-hand side we use the estimate

$$
(f, u) \leq(A u, u)+\frac{1}{4}\left(A^{-1} f, f\right) .
$$

This yields

$$
\frac{d}{d t}\|u\|_{B}^{2} \leq \frac{1}{2}\|f\|_{A^{-1}}^{2} .
$$

Taking into account Gronwall's inequality we obtain the following a priori estimate for the solution of problem (1.1), (1.2):

$$
\|u(t)\|_{B}^{2} \leq\left\|u^{0}\right\|_{B}^{2}+\frac{1}{2} \int_{0}^{t}\|f(s)\|_{A^{-1}}^{2} d s,
$$

which expresses the stability of the solution with respect to the initial data and right-hand side.

Standard additive difference schemes are characterized by decomposition (splitting) of the operator $A$ onto the sum of operators of a simpler structure. For example, we assume that for operator $A$ we have the following additive representation:

$$
A=\sum_{\alpha=1}^{p} A_{\alpha}, \quad A_{\alpha}=A_{\alpha}^{*} \geq 0, \quad \alpha=1,2, \ldots, p .
$$

Additive difference schemes are based on the basis of (1.4), where the problem is decomposed into $p$ subproblems. The transition from time level $t^{n}$ to the next level $t^{n+1}=t^{n}+\tau$, where $\tau>0$ is the time step and $y^{n}=y\left(t^{n}\right), t^{n}=n \tau, n=0,1, \ldots$, is associated with solving problems for individual operators $A_{\alpha}, \alpha=1,2, \ldots, p$ in additive decomposition (1.4).

The subject we consider will be another case. In a number of problems the computational complexity is not associated with operator $A$, but with operator $B$ 
at the derivatives in time. In this case, to decrease the computational complexity of problem (1.1), (1.2) we employ the additive representation

$$
B=\sum_{\alpha=1}^{p} B_{\alpha}, \quad B_{\alpha}=B_{\alpha}^{*}>0, \quad \alpha=1,2, \ldots, p .
$$

instead of (1.4). The transition to a new time level is connected with the solution of some auxiliary Cauchy problems for equations

$$
B_{\alpha} \frac{d u_{\alpha}}{d t}+A u_{\alpha}=f_{\alpha}(t), \quad t>0, \quad \alpha=1,2, \ldots, p
$$

for one or another selection of $f_{\alpha}(t), \alpha=1,2, \ldots, p$, with specified appropriate initial conditions.

\section{VECTOR PROBLEM}

By definition, put $\mathbf{u}=\left\{u_{1}, u_{2}, \ldots, u_{p}\right\}$. Each individual component is defined as the solution of similar problems

$$
\begin{gathered}
\sum_{\beta=1}^{p} B_{\beta} \frac{d u_{\beta}}{d t}+A u_{\alpha}=f(t), \quad t>0, \\
u_{\alpha}(0)=u^{0}, \quad \alpha=1,2, \ldots, p .
\end{gathered}
$$

Here is the simplest coordinatewise estimate for the stability of the solution. Subtracting one equation from another, we get

$$
A\left(u_{\alpha}-u_{\alpha-1}\right)=0, \quad \alpha=2,3, \ldots, p .
$$

Taking into account the positivity of operator $A$, this gives

$$
u_{\alpha}=u_{\alpha-1}, \quad \alpha=2,3, \ldots, p .
$$

For separate component $u_{\alpha}$ we obtain the same equation as for $u$ :

$$
\sum_{\beta=1}^{p} B_{\beta} \frac{d u_{\alpha}}{d t}+A u_{\alpha}=f(t), \quad t>0, \quad \alpha=1,2, \ldots, p .
$$

For the same reason, there are a priori estimates

$$
\left\|u_{\alpha}(t)\right\|_{B}^{2} \leq\left\|u^{0}\right\|_{B}^{2}+\frac{1}{2} \int_{0}^{t}\|f(s)\|_{A^{-1}}^{2} d s, \quad \alpha=1,2, \ldots, p .
$$

It follows that

$$
u_{\alpha}(t)=u(t), \quad t>0, \quad \alpha=1,2, \ldots, p .
$$

Therefore, as the solution of the original problem (1.1), (1.2) we can take any component of the vector $\mathbf{u}(t)$.

For the vector evolutionary problem we can obtain a priori estimates for the vector $\mathbf{u}$, considering the problem in Hilbert space $\mathbf{H}=H^{p}$ with the scalar product

$$
(\mathbf{u}, \mathbf{v})=\sum_{\alpha=1}^{p}\left(u_{\alpha}, v_{\alpha}\right)
$$

This technique is used, for example, in [14] when considering additive schemes with splitting (1.4). 
We rewrite equations (2.1) in the form

$$
B_{\alpha} A^{-1} \sum_{\beta=1}^{p} B_{\beta} \frac{d u_{\beta}}{d t}+B_{\alpha} u_{\alpha}=\tilde{f}_{\alpha}(t), \quad t>0, \quad \alpha=1,2, \ldots, p,
$$

where $\tilde{f}_{\alpha}=B_{\alpha} A^{-1} f$. This allows us to write the system of equations in vector form:

$$
\mathbf{C} \frac{d \mathbf{u}}{d t}+\mathbf{D u}=\tilde{\mathbf{f}}
$$

Operator matrix $\mathbf{C}$ and $\mathbf{D}$ have the form

$$
\begin{gathered}
\mathbf{C}=\left\{C_{\alpha \beta}\right\}, \quad C_{\alpha \beta}=B_{\alpha} A^{-1} B_{\beta}, \\
\mathbf{D}=\left\{D_{\alpha \beta}\right\}, \quad D_{\alpha \beta}=B_{\alpha} \delta_{\alpha \beta}, \quad \alpha, \beta=1,2, \ldots, p,
\end{gathered}
$$

where $\delta_{\alpha \beta}$ is the Kronecker delta. Equation (2.4) is supplemented by the initial condition

$$
\mathbf{u}(0)=\mathbf{u}^{0}
$$

The principal advantage of notation (2.4) results from the fact that

$$
\mathbf{C}=\mathbf{C}^{*} \geq 0, \quad \mathbf{D}=\mathbf{D}^{*}>0
$$

in $\mathbf{H}$.

Here is an a priori estimate for the solution of vector problem (2.4)-(2.6). This estimate, on the one hand, is more complicated than (2.3) and, on the other hand, we will use it as a guide in considering the operator-difference schemes.

Multiplying both sides of (2.4) scalarly in $\mathbf{H}$ by $d \mathbf{u} / d t$, we get

$$
\left(\mathbf{C} \frac{d \mathbf{u}}{d t}, \frac{d \mathbf{u}}{d t}\right)+\frac{1}{2} \frac{d}{d t}(\mathbf{D u}, \mathbf{u})=\left(\tilde{\mathbf{f}}, \frac{d \mathbf{u}}{d t}\right) .
$$

Taking into account (2.5), we obtain

$$
\left(\mathbf{C} \frac{d \mathbf{u}}{d t}, \frac{d \mathbf{u}}{d t}\right)=\left(A^{-1} \sum_{\beta=1}^{p} B_{\beta} u_{\beta}, \sum_{\beta=1}^{p} B_{\beta} u_{\beta}\right),
$$

and for the right-hand side of (2.7) we have

$$
\left(\tilde{\mathbf{f}}, \frac{d \mathbf{u}}{d t}\right)=\left(A^{-1} f, \sum_{\beta=1}^{p} B_{\beta} u_{\beta}\right) \leq\left(\mathbf{C} \frac{d \mathbf{u}}{d t}, \frac{d \mathbf{u}}{d t}\right)+\frac{1}{4}\left(A^{-1} f, f\right) .
$$

Similar to (1.3), (2.3), from (2.7), (2.8) it follows that the estimate holds:

$$
\|\mathbf{u}\|_{\mathbf{D}}^{2} \leq\left\|\mathbf{u}^{0}\right\|_{\mathbf{D}}^{2}+\frac{1}{2} \int_{0}^{t}\|f(s)\|_{A^{-1}}^{2} d s
$$

Taking into account (2.5), we have

$$
\|\mathbf{u}\|_{\mathbf{D}}^{2}=\sum_{\alpha=1}^{p}\left(B_{\alpha} u_{\alpha}, u_{\alpha}\right)
$$

Thus, estimate (2.9) can be considered along with (2.3) as the vector analogue of estimate (1.3). Taking into account (1.5), estimate (2.9) gives the stability of any individual component of vector $\mathbf{u}(t)$. 


\section{Additive Vector SCHEMES}

Splitting schemes for the approximate solution of (1.1), (1.2), (1.5) will be constructed on the basis of usual schemes with weights for vector problem (2.1), (2.2).

The standard two-level scheme with weights for problem (1.1), (1.2) has the form

$$
B \frac{y^{n+1}-y^{n}}{\tau}+A\left(\sigma y^{n+1}+(1-\sigma) y^{n}\right)=\varphi^{n}, \quad n=0,1, \ldots,
$$

where, for example,

$$
\varphi^{n}=f\left(\sigma t^{n+1}+(1-\sigma) t^{n}\right),
$$

and $\sigma$ is a weight parameter (usually $0 \leq \sigma \leq 1$ ).

In the general theory of operator-difference schemes stability developed by A.A. Samarskii [10 12], the exact (not improvable) stability criteria for two-level and three-level operator-difference schemes in various norms were obtained. They can be used directly in the study of schemes with weights (3.1). Here is a typical result.

Theorem 3.1. If $\sigma \geq 1 / 2$, then operator-difference scheme (3.1) is absolutely stable in $H_{B}$ and for the difference solution the level-wise estimate is valid:

$$
\left\|y^{n+1}\right\|_{B}^{2} \leq\left\|y^{n}\right\|_{B}^{2}+\frac{\tau}{2}\left\|\varphi^{n}\right\|_{A^{-1}}^{2} .
$$

Proof. By definition, put

$$
y^{\sigma(n)}=\sigma y^{n+1}+(1-\sigma) y^{n}=\frac{1}{2}\left(y^{n+1}+y^{n}\right)+\tau\left(\sigma-\frac{1}{2}\right) \frac{y^{n+1}-y^{n}}{\tau} .
$$

Multiplying both sides of (3.1) by $y^{\sigma(n)}$ scalarly in $H$, we get

$$
\begin{gathered}
\frac{1}{2 \tau}\left(B\left(y^{n+1}-y^{n}\right), y^{n+1}+y^{n}\right)+ \\
\tau\left(\sigma-\frac{1}{2}\right)\left(B \frac{y^{n+1}-y^{n}}{\tau}, \frac{y^{n+1}-y^{n}}{\tau}\right)+\left(A y^{\sigma(n)}, y^{\sigma(n)}\right)=\left(\varphi^{n}, y^{\sigma(n)}\right) .
\end{gathered}
$$

For the right-hand side we use the estimate

$$
\left(\varphi^{n}, y^{\sigma(n)}\right) \leq\left(A y^{\sigma(n)}, y^{\sigma(n)}\right)+\frac{1}{4}\left(A^{-1} \varphi^{n}, \varphi^{n}\right) .
$$

If $\sigma \geq 1 / 2$, we obtain the desired estimate (3.2) for the stability of the numerical solution with respect to the initial data and the right-hand side, which is the grid analog of estimate (1.3) for the solution of problem (1.1), (1.2). This concludes the proof.

To solve vector problem (2.1), (2.2) we apply the following difference scheme:

$$
\begin{gathered}
B_{\alpha}\left(\theta \frac{y_{\alpha}^{n+1}-y_{\alpha}^{n}}{\tau}+(1-\theta) \frac{y_{\alpha}^{n}-y_{\alpha}^{n-1}}{\tau}\right)+ \\
\sum_{\alpha \neq \beta=1}^{p} B_{\beta} \frac{y_{\beta}^{n}-y_{\beta}^{n-1}}{\tau}+A\left(\sigma y_{\alpha}^{n+1}+(1-2 \sigma) y_{\alpha}^{n}+\sigma y_{\alpha}^{n-1}\right)=\varphi^{n} \\
n=0,1, \ldots, \quad \alpha=1,2, \ldots, p .
\end{gathered}
$$

Unlike (3.1) scheme (3.3) is a three-level one and has two weight factors $\theta$ and $\sigma$.

Numerical implementation of scheme (3.3) is associated with solving sequential grid problems

$$
\left(\theta B_{\alpha}^{n}+\sigma \tau A\right) y_{\alpha}^{n+1}=\chi_{\alpha}^{n}, \quad \alpha=1,2, \ldots, p,
$$


with transition from time level $t^{n}$ to new time level $t^{n+1}$. For vector additive scheme (3.3) it is possible to implement a parallel organization of computations an independent calculation of the individual components.

Using notation (2.5), we write operator-difference scheme (3.3) in the vector form

$$
\begin{gathered}
\theta \mathbf{G} \frac{\mathbf{y}^{n+1}-2 \mathbf{y}^{n}+\mathbf{y}^{n-1}}{\tau}+ \\
\mathbf{C} \frac{\mathbf{y}^{n}-\mathbf{y}^{n-1}}{\tau}+\mathbf{D}\left(\sigma \mathbf{y}^{n+1}+(1-2 \sigma) \mathbf{y}^{n}+\sigma \mathbf{y}^{n-1}\right)=\mathbf{g}^{n},
\end{gathered}
$$

where

$$
\begin{gathered}
\mathbf{G}=\left\{G_{\alpha \beta}\right\}, \quad G_{\alpha \beta}=B_{\alpha} A^{-1} B_{\alpha} \delta_{\alpha \beta}, \\
\mathbf{g}^{n}=\left\{g_{\alpha}^{n}\right\}, \quad g_{\alpha}^{n}=B_{\alpha} A^{-1} \varphi^{n}, \quad \alpha, \beta=1,2, \ldots, p .
\end{gathered}
$$

Thus, in (3.4) operator $\mathbf{G}=\mathbf{G}^{*}>0$.

Taking into account that

$$
\begin{gathered}
\frac{\mathbf{y}^{n}-\mathbf{y}^{n-1}}{\tau}=\frac{\mathbf{y}^{n+1}-\mathbf{y}^{n-1}}{2 \tau}-\frac{\mathbf{y}^{n+1}-2 \mathbf{y}^{n+1}+\mathbf{y}^{n-1}}{2 \tau}, \\
\sigma \mathbf{y}^{n+1}+(1-2 \sigma) \mathbf{y}^{n}+\sigma \mathbf{y}^{n-1}= \\
\left(\sigma-\frac{1}{4}\right)\left(\mathbf{y}^{n+1}-2 \mathbf{y}^{n+1}+\mathbf{y}^{n-1}\right)+\frac{1}{4}\left(\mathbf{y}^{n+1}+2 \mathbf{y}^{n+1}+\mathbf{y}^{n-1}\right),
\end{gathered}
$$

rewrite (3.4) in the form

where

$$
\begin{gathered}
\mathbf{C} \frac{\mathbf{y}^{n+1}-\mathbf{y}^{n-1}}{2 \tau}+\mathbf{R} \frac{\mathbf{y}^{n+1}-2 \mathbf{y}^{n+1}+\mathbf{y}^{n-1}}{\tau}+ \\
\frac{1}{4} \mathbf{D}\left(\mathbf{y}^{n+1}+2 \mathbf{y}^{n+1}+\mathbf{y}^{n-1}\right)=\mathbf{g}^{n},
\end{gathered}
$$

Let

$$
\mathbf{R}=\theta \mathbf{G}-\frac{1}{2} \mathbf{C}+\tau\left(\sigma-\frac{1}{4}\right) \mathbf{D} .
$$

and rewrite (3.5) in the form

$$
\mathbf{C} \frac{\mathbf{w}^{n+1}+\mathbf{w}^{n}}{2 \tau}+\mathbf{R} \frac{\mathbf{w}^{n+1}-\mathbf{w}^{n}}{\tau}+\frac{1}{2} \mathbf{D}\left(\mathbf{v}^{n+1}+\mathbf{y}^{n}\right)=\mathbf{g}^{n} .
$$

Multiplying both sides of (3.6) scalarly by

$$
2\left(\mathbf{v}^{n+1}-\mathbf{v}^{n}\right)=\mathbf{w}^{n+1}+\mathbf{w}^{n},
$$

we get the equality

$$
\begin{gathered}
\frac{1}{2 \tau}\left(\mathbf{C}\left(\mathbf{w}^{n+1}+\mathbf{w}^{n}\right), \mathbf{w}^{n+1}+\mathbf{w}^{n}\right)+\frac{1}{\tau}\left(\mathbf{R}\left(\mathbf{w}^{n+1}-\mathbf{w}^{n}\right), \mathbf{w}^{n+1}+\mathbf{w}^{n}\right)+ \\
\left(\mathbf{D}\left(\mathbf{v}^{n+1}+\mathbf{v}^{n}\right), \mathbf{v}^{n+1}-\mathbf{v}^{n}\right)=\left(\mathbf{g}^{n}, \mathbf{w}^{n+1}+\mathbf{w}^{n}\right) .
\end{gathered}
$$

Similar to (2.8), we have

$$
\left(\mathbf{g}^{n}, \mathbf{w}^{n+1}+\mathbf{w}^{n}\right) \leq \frac{1}{2 \tau}\left(\mathbf{C}\left(\mathbf{w}^{n+1}+\mathbf{w}^{n}\right), \mathbf{w}^{n+1}+\mathbf{w}^{n}\right)+\frac{\tau}{2}\left(A^{-1} \varphi^{n}, \varphi^{n}\right) .
$$

With this in mind, from (3.7) it follows that

$$
\mathcal{E}_{n+1} \leq \mathcal{E}_{n}+\frac{\tau}{2}\left(A^{-1} \varphi^{n}, \varphi^{n}\right)
$$


where

$$
\mathcal{E}_{n}=\left(\mathbf{D} \mathbf{v}^{n}, \mathbf{v}^{n}\right)+\frac{1}{\tau}\left(\mathbf{R} \mathbf{w}^{n}, \mathbf{w}^{n}\right) .
$$

We formulate the conditions under which the value of $\mathcal{E}_{n}$ determines the square of the norm of the difference solution. By virtue of the positivity of operator $\mathbf{D}$ it is sufficient to require non-negativity of operator $\mathbf{R}$.

For the energy of operators $\mathbf{C}$ and $\mathbf{G}$, the following coordinatewise representation holds:

$$
\begin{gathered}
(\mathbf{C u}, \mathbf{u})=\left(A^{-1} \sum_{\alpha=1}^{p} B_{\alpha} u_{\alpha}, \sum_{\alpha=1}^{p} B_{\alpha} u_{\alpha}\right), \\
(\mathbf{G u}, \mathbf{u})=\sum_{\alpha=1}^{p}\left(A^{-1} B_{\alpha} u_{\alpha}, B_{\alpha} u_{\alpha}\right) .
\end{gathered}
$$

Considering

$$
\begin{aligned}
& \left(A^{-1} \sum_{\alpha=1}^{p} B_{\alpha} u_{\alpha}, \sum_{\alpha=1}^{p} B_{\alpha} u_{\alpha}\right)=\left(\sum_{\alpha=1}^{p}\left(A^{-1 / 2} B_{\alpha} u_{\alpha}\right)^{2}, 1\right) \\
& \leq p \sum_{\alpha=1}^{p}\left(\left(A^{-1 / 2} B_{\alpha} u_{\alpha}\right)^{2}, 1\right)=p \sum_{\alpha=1}^{p}\left(A^{-1} B_{\alpha} u_{\alpha}, B_{\alpha} u_{\alpha}\right)
\end{aligned}
$$

we get

$$
\mathbf{C} \leq p \mathbf{G}
$$

Therefore, at $\sigma \geq 1 / 4$ and $\theta \geq p / 2$ it holds that $\mathbf{R} \geq 0$. We have thus proved the following assertion.

Theorem 3.2. If $\sigma \geq 1 / 4$ and $\theta \geq p / 2$, then operator $\mathbf{R} \geq 0$ in $\mathbf{H}$, an additive vector scheme (3.3) is absolutely stable, and the difference solution holds a priori for estimate (3.8) with

$$
\mathcal{E}_{n}=\left\|\frac{\mathbf{y}^{n}+\mathbf{y}^{n-1}}{2}\right\|_{\mathbf{D}}+\frac{1}{\tau}\left(\mathbf{R}\left(\mathbf{y}^{n}-\mathbf{y}^{n-1}\right), \mathbf{y}^{n}-\mathbf{y}^{n-1}\right) .
$$

Proved a priori estimate (3.8) guarantees the stability of the difference solution in the half-integer time levels (for $\mathbf{v}^{n}$ ) and is the difference analogue for estimate (2.9).

\section{Generalizations}

We note some of the key research areas that focus on the synthesis and development of the obtained results.

On the basis of a priori estimate (3.8), we obtain the convergence of the solution of the difference problem (3.3) to the solution of the differential problem (1.1), (1.2) with the first order of $\tau$. In the standard way [10] we consider the problem for the truncation error using a particular scheme for finding the solution at the first time level. 
Instead of (3.3) we can use another additive scheme. In the class of vector additive schemes, in particular, special attention should be given to the scheme

$$
\begin{gathered}
\sum_{\beta=1}^{\alpha} B_{\beta} \frac{y_{\beta}^{n+1}-y_{\beta}^{n}}{\tau}+\sum_{\beta=\alpha+1}^{p} B_{\beta} \frac{y_{\beta}^{n}-y_{\beta}^{n-1}}{\tau}+ \\
A\left(\sigma y_{\alpha}^{n+1}+(1-2 \sigma) y_{\alpha}^{n}+\sigma y_{\alpha}^{n-1}\right)=\varphi^{n} \\
n=0,1, \ldots, \quad \alpha=1,2, \ldots, p .
\end{gathered}
$$

In this case, the time derivative of the several components of the vector solution relates to the upper time-level. Such vector additive schemes are widely used [1, 13. with usual decomposition (1.4).

Some resources are available when considering more general problems than (1.1), (1.2), (1.5). In our study we restricted ourselves to the simplest problems, where operators $A, B$ and the components of splitting $B_{\alpha}, \alpha=1,2, \ldots, p$ are constant selfadjoint and positive in finite Hilbert space $H$. These restrictions can be removed in some cases, by analogy with the theory of additive schemes for problems (1.1), (1.2) with the usual splitting of (1.5), considering, for example, problems with not self-adjoint operators, and problems with operator factors [12,14.

In terms of generalizing the results, the greatest interest is to construct the additive operator-difference schemes for solving the Cauchy problem for evolutionary equation (1.1) in the splitting of both operator $A$ and operator $B$ - for the problem (1.1), (1.2), (1.4), (1.5). In this case the transition to the new time level is based on solving a sequence of problems for equations

$$
B_{\alpha} \frac{d u_{\alpha}}{d t}+A_{\alpha} u_{\alpha}=f_{\alpha}(t), \quad t>0, \quad \alpha=1,2, \ldots, p,
$$

with appropriate initial conditions.

\section{REFERENCES}

[1] V.N. Abrashin, A variant of the method of variable directions for the solution of multidimensional problems of mathematical-physics, Differ. Equations 26 (1990), no. 2, 243-250. MR.1050397 (91c:35003)

[2] J. Douglas and H.H. Rachford, On the numerical solution of heat conduction problems in two and three space variables, Trans. Amer. Math. Soc. 82 (1956), 421-439. MR0084194 $(18: 827 \mathrm{f})$

[3] R.E. Ewing, Numerical solution of Sobolev partial differential equations, SIAM Journal on Numerical Analysis 12 (1975), no. 3, 345-363. MR0395265 (52:16062)

[4] _ Time-stepping Galerkin methods for nonlinear Sobolev partial differential equations, SIAM Journal on Numerical Analysis 15 (1978), no. 6, 1125-1150. MR512687 (80b:65136)

[5] D.G. Gordeziani and G.V. Meladze, Simulation of the third boundary value problem for multidimensional parabolic equations in an arbitrary domain by one-dimensional equations, Computational Mathematics and Mathematical Physics 14 (1974), no. 1, 249-253. MR0400734 (53:4564)

[6] T. Mathew, Domain decomposition methods for the numerical solution of partial differential equations, Lecture Notes in Computational Science and Engineering 61. Berlin: Springer. xiv+764 pp., 2008. MR2445659 (2010b:65006)

[7] G.I. Marchuk, Splitting and alternating direction methods, Handbook of numerical analysis, Vol. I, 1990, 197. MR1039325

[8] D.W. Peaceman and H.H. Rachford, The numerical solution of parabolic and elliptic differential equations, J. Soc. Ind. Appl. Math. 3 (1955), 28-41. MR0071874(17:196d)

[9] A.A. Samarskii, Regularization of difference schemes, Computational Mathematics and Mathematical Physics 7 (1967), no. 1, 79-120. 
[10] _ The theory of difference schemes, Pure and Applied Mathematics, 240. Marcel Dekker, Inc., New York, xviii+761 pp. MR1818323 (2002c:65003)

[11] A.A. Samarskii and A.V. Gulin, Stability of difference schemes, Moscow: URSS. 384 p., 2005 (Russian). MR2263771(2008a:65001)

[12] A.A. Samarskii, P.P. Matus, and P.N. Vabishchevich, Difference schemes with operator factors, Mathematics and its Applications (Dordrecht). 546. Dordrecht: Kluwer Academic Publishers. $\mathrm{x}+384$ pp., 2002. MR.1950844 (2003k:65095)

[13] _ Stability of vector additive schemes, Doklady Mathematics 58 (1998), no. 1, 133-135.

[14] A.A. Samarskii and P.N. Vabishchevich, Additive schemes for problems of mathematical physics, Moscow: Nauka. 320 p., 1999 (Russian). MR.1714030(2000e:65004)

[15] _ Regularized additive full approximation schemes, Doklady Mathematics 57 (1998), $83-86$.

[16] S.L. Sobolev, Some new problems in mathematical physics, Izv. Akad. Nauk SSSR Ser. Mat 18 (1954), 3-50.

[17] R.E. Showalter, Partial differential equations of Sobolev-Galpern type, Pacific J. Math 31 (1969), no. 3, 787-793. MR0252870 (40:6085)

[18] R.E. Showalter and T.W. Ting, Pseudoparabolic partial differential equations, Siam J. Math. Anal 1 (1970), no. 1, 1-26. MR0437936 (55:10857)

[19] A.G. Sveshnikov, A.B. Al'shin, M.O. Korpusov, and Y.D. Pletner, Linear and nonlinear equations of Sobolev type, Moscow: Fizmatlit. 734 p., 2007 (Russian).

[20] P.N. Vabishchevich, Vector additive difference schemes for first-order evolution equations, Computational Mathematics and Mathematical Physics 36 (1996), no. 3, 317-322. MR1382641

[21] _ Regularized additive operator-difference schemes, Computational Mathematics and Mathematical Physics 50 (2010), no. 3, 428-436. MR.2681922 (2011f:65134)

$[22]$ _ Domain decomposition methods with overlapping subdomains for the time-dependent problems of mathematical physics, Computational Methods in Applied Mathematics 8 (2008), 393-405. MR2604752

[23] N.N. Yanenko, The method of fractional steps. The solution of problems of mathematical physics in several variables, Berlin-Heidelberg-New York: Springer Verlag, VIII, 160 p., 1971. MR0307493(46:6613)

Keldysh Institute of Applied Mathematics, Russian Academy of Sciences, 4 Miusskaya SQ., 125047 Moscow, Russia

E-mail address: vabishchevich@gmail.com 